\title{
On the Derivative of a Polynomial with Prescribed Zeros
}

\author{
Vinay K. Jain
}

ABSTRACT: For a polynomial $p(z)=a_{n} \prod_{t=1}^{n}\left(z-z_{t}\right)$ of degree $n$ having all its zeros in $|z| \leq K, K \geq 1$ it is known that

$$
\max _{|z|=1}\left|p^{\prime}(z)\right| \geq \frac{2}{1+K^{n}}\left\{\sum_{t=1}^{n} \frac{K}{K+\left|z_{t}\right|}\right\} \max _{|z|=1}|p(z)| .
$$

By assuming a possible zero of order $m, 0 \leq m \leq n-4$, at $z=0$, of $p(z)$ for $n \geq k+m+1$ with integer $k \geq 3$ we have obtained a new refinement of the known result. 